\title{
Mobile Mediated Communication: Emoji Usage and Play Frame
}

\author{
Qi Su and Xiaoxiao Chen*
}

\author{
School of English for International Business, Guangdong University of Foreign Studies \\ *Corresponding author.Email: gracekot@qq.com
}

\begin{abstract}
Emoji has already become a ubiquitous language for Mobile Mediated Communication (MMC) worldwide. And emoji usage has many implications from text messaging to social interaction. It helps users convey emotional information via nonverbal cues. Although prior sentiment analyses provide clear pictures of emoji usage, the expression intensity of an emoji varies from one to another in different speech community. Besides, the communicative functions emoji serves might depend on mediated platforms. Therefore, present study aims to describe how college students apply QQ emojis to engage in their social networks. Based on the collected chatting records, QQ emojis displayed prominent appeal and courtesy functions in push, notice and questionnaire request and the expression intensity proportionately correlate with the use frequency. Finally, the notion of play interprets emoji usage, arguing that the effects emoji brings, is as much a result of users' preference as it is born to satisfy the needs of communicating visually in telephony.
\end{abstract}

Keywords: $M M C$, emoji usage, the notion of play, $Q Q$ emoji, college student

\section{INTRODUCTION}

In early days, emoticons are often discussed in the field of Computer Mediated Communication (CMC). However, mediated communication is heavily driven by socio-technological forces and cellphone is another major device for the infiltration of emoji. As an emotional icon, emoji conveys information through nonverbal cues. As an arbitrary symbol, emoji conveys information in contexts. Clearly, the spread of emoji harbors a broad range of implications. While emoji users, an implied facet, is easy to be overlooked in literature as prior studies are keen for demystifying the true nature of emoji. Although empirical studies have proved the contribution of emoji in enhancing online communicative quality [1], concerns over emoji usage are growing. To make a critical stance while maintaining involvement is needed.

Mediated platform is a major independent variable in emoji studies, while target groups tend to be the young as they feel natural to communicate electronically. Present study follows a functional approach to explore how college students use QQ emojis to engage in their social activities. Specially, what makes a good emoji and what functions it serves to communicate effectively in messages. Sentiment analysis [2] is, therefore, pertinent as it analyzes emotions of users based on polarity from a digital text. What's more, play frame and playfulness shed light on emoji usage. The former suggests how users play emojis matters, resulting in uneven expression intensities. While the latter explains why college students prefer popular emojis. And playfulness is so hard to control in a pan-entertainment internet community that would probably generate another limitation of emoji usage except contextual ambiguity caused by semiotic arbitrariness.

\section{LITERATURE REVIEW}

Emoji, as a visual sign of emotion, was introduced by Japanese engineer Shigetaka Kurita in 1998, aiming to address the data-consuming problem when people sent pictures back and forth on the mobile phone.

\subsection{Mobile Mediated Communication}

Previous research consent to explore emoji within the scope of CMC [3] which has previewed mobile telephony. And the move towards a media integration would pose a question on the position of those CMC studies. MMC is more suitable for emoji studies as technological advancements always lead tremendous change in social life. And a critical area for social science is how emojis in various APPs impact communication quality. The carried substantial proportion of nonverbal cues are thus, 
the target of many researchers. Indeed, emojis afford users more choices to overcome linguistic limitations during online chatting. However, there are claims that emoji is not language because they are not rule governed. Besides, nonverbal cues produce multiple meanings which often generate ambiguity [4].

\subsection{Emoji as a linguistic sign}

In essence, emoji (signifier) is a meaningless symbol endowed with a denotation (signified), then the emoji can represent certain of information in context, according to Saussure. And questions like what does the meaning an emoji express and how an emoji connects with its denotation usually come from semiotics. Emoji, as a linguistic sign in digital space, is likely to cause communicating barriers between sender and receiver because of referential arbitrariness. In speech acts, emoji belongs to expressive, which is used to describe speaker's inner world. Emoji is also a good anchor attached to depict how emojis make sense in conversation from abstract pictorial symbols to concrete semantic concepts [5]. As a visualized output of emotion, the formation and understanding process of emoji involves the encoding the decoding between physical reality and virtual internet. Emoji studies, from the linguistic perspective, start from a micro level and focus on the symbolic meaning, which contributes to its publicity and development. For one thing, not only has our visual vocabulary of emotional module been enriched, but emojis continue to update and expand. For another, it is no longer an auxiliary of language, we add emoji social attribute in digital network. It may thus, be analytically or theoretically convenient to exclude social factors when conducting discourse analysis, but it always runs the risk of missing the point: the mismatch between users' attempts and emojis' denotations in conversation.

\subsection{Emoji as a social tool}

In Kinesics, emoji represents human gestures and movements within a social context. And the famous "7$38-55$ rule" (mutual understanding $=38 \%$ intonation + $55 \%$ facial expressions $+7 \%$ language) is welcomed by practitioners of the field to account for the behavoir of emoji usage. While Dramaturgy compares emoji usage with theatrical performance. In the play, those APPs is the theatre where user is the trouper and emoji is the prop. Related research observe how they manage their virtual self-image with the help of emoji. As for popularity, both Diffusion and Innovation Theory and Memetics have explanations. Within internet language, emoji can be easily assimilated, retained, expressed and transformed. Gradually, emoji subcultures generate. However, an enrichment in cultural meaning implies potential incomprehension between insider and outsider. Emoji studies, from sociological perspective, start from a macro level and focus on functions and impacts. And experimental variables could be gender, age or mediated platform. As for theme, personality assessment [6] and interpersonal relationship [7] could be conducted via "emoji identity". Sentiment analysis accounts for the majority though statistical deviation seems to be unavoidable. Further, to sentimentally quantify all the observed emojis is persuasive but we question those unpopular emojis with relatively high emotional vectors.

\subsection{The notion of play}

Play is an activity in which we engage only because we want to, not because we feel we must [8]. The notion of play in human activity is the core of playfulness in social psychology which could be easily generalized to expound social interaction. And playfulness could be defined as a sense of enjoyment brought by social software. In MMC, emoji usage is a kind of play behavior. Users manage their social networks with the goal of seeking for fun. However, to entertain is not the sole function of emoji, though pan-entertainment [9] channels current mainstream internet culture. The notion of play is also the most salient framework of explanation for most of the social interaction where recreation is oriented in chatting. Play frame [10], shown as mental schemata, is a dynamic construct and available for interlocutors to structure their experiences at any time. Since it is not the result of a conscious rationalization, it can be applied to address emoji usage for the following reasons. Firstly, college students share a common ground on how to encode and decode emojis. Secondly, college students are not fully aware of play frame that emerges from discourse context. Thirdly, play frame drives emojis to instantiate some stylistic patterns or linguistic features though it is an abstract concept.

\section{RESEARCH METHOD}

In order to fill the gaps, a descriptive analysis was conducted based on chatting records $(2018.5-2018.10)$ of 3 groups (738 college students), of which 329 types of QQ emojis (Tencent QQ 7.3.5) were spotlighted. Especially: a) to what extend can an emoji help college students to express one type of basic emotions in MMC? b) what are the communicative functions of emojis in dialog box when college students engage in their social networks? With the help of Sketch Engine, 2,424 pieces of messages containing 689 emojis in 95 types were collected. Due to privacy and ethical consideration, only metadata for research was extracted and presented.

\section{RESULTS}

$17.24 \%$ of messages contained at least one emoji and "mono-emoji + word" was the most popular emoji usage pattern. Based on sentiment polarity and emotion classification [11], the collected emojis were classified into six groups: joy, sad, surprise, anger, disgust and fear 
(data nonexistent). Positive and neutral emojis accounted for $86.46 \%$ of the total data.

\subsection{Emotion expression intensity}

Emoji Sentiment Ranking [12] helped create a fivedimension vectorial equation to measure expression intensity of classified emojis. If $\mathrm{N}\left(\mathrm{e}_{\mathrm{i}}, \mathrm{E}_{\mathrm{n}}\right)$ stated the frequency of the message with both the i-th emoji and the n-th kind of emotion, then Equation (1) represented the expression intensity of the i-th emoji towards the n-th kind of emotion.

$C_{e_{i n}}$ in $=\frac{N\left(e_{i} E_{n}\right)}{\sum_{n=1}^{5} N\left(e_{i}, E_{n}\right)} \times \lg \left(n\left(e_{i}\right)\right)=\lg \left(n\left(e_{i}\right)\right)$

Table 1. Sentimental Vector of the Top 10 Emojis

\begin{tabular}{|c|c|c|c|c|c|c|}
\hline \multicolumn{2}{|c|}{ Sentiment polarity } & \multirow{2}{*}{$\begin{array}{c}\text { Positive } \\
\text { Joy }\end{array}$} & \multirow{2}{*}{$\begin{array}{l}\text { Neutral } \\
\text { Surprise }\end{array}$} & \multicolumn{3}{|c|}{ Negative } \\
\hline Emoji & $n\left(e_{i}, E_{n}\right)$ & & & Sad & Anger & Disgust \\
\hline$\Leftrightarrow$ & 57 & 1.9542 & 1.0792 & 1.9494 & 0.7782 & 0 \\
\hline होग & 47 & 1.6902 & & 0 & 0 & \\
\hline 2 & 24 & 1.6812 & & 0 & & \\
\hline 3 & 7 & & 0.6990 & 0.8451 & 0 & \\
\hline 3 & 23 & 1.4150 & 0.3010 & 1.4150 & 0.4771 & \\
\hline (3) & 19 & 1.4624 & & & & \\
\hline$\bullet$ & 10 & 1.3424 & & & & \\
\hline 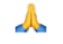 & 10 & 1.1139 & & 0.4771 & & \\
\hline -60 & 8 & & & 1.1139 & 0 & \\
\hline$(2-3)$ & 7 & 1.1139 & & & & \\
\hline
\end{tabular}

Emojis with relatively high vectors were shown in yellow blank. $80 \%$ of emojis had well explained joy, and $40 \%$ of emojis finely said sad emotion. Inspired by 2.3 , we compared $\mathrm{N}$ with the frequencies of top 10 most used emojis (hereafter $\mathrm{T}$ ). When $\mathrm{N}=0.6004 \mathrm{~T}$, the emojis in red blank had a better vector or a more accurate expression intensity towards the corresponding emotions.

\subsection{Three Communicative functions}

There was a convergence among long messages ( $>100$ characters) which could be categorized into three groups: push, notice, and questionnaire request. Emojis in those contexts also performed three similar functions - Appeal, Courtesy and Emotion - of which courtesy was the most prominent one. As shown in Table 2, "rose" and "folded hands" was commonly used to express "thanks" and "please" respectively. And "OK", indicating "no problem" or "finished", was a typical replay to questionnaire request. Whereas face-related emojis tended to arise in push and no emojis had been identified to express emotion in notice and questionnaire request. Besides, as what 2.4 suggested, long messages bore textual resemblances with the correspondence which led the emojis to present some features in terms of position and format. Specifically, those emoji showed behind words in general, especially "courtesy emojis", which were also prone to be in multiple form. While "appeal emojis" were usually placed at the beginning and facial emojis inclined to be in single form.

Table 2. The Position and Format of Emojis

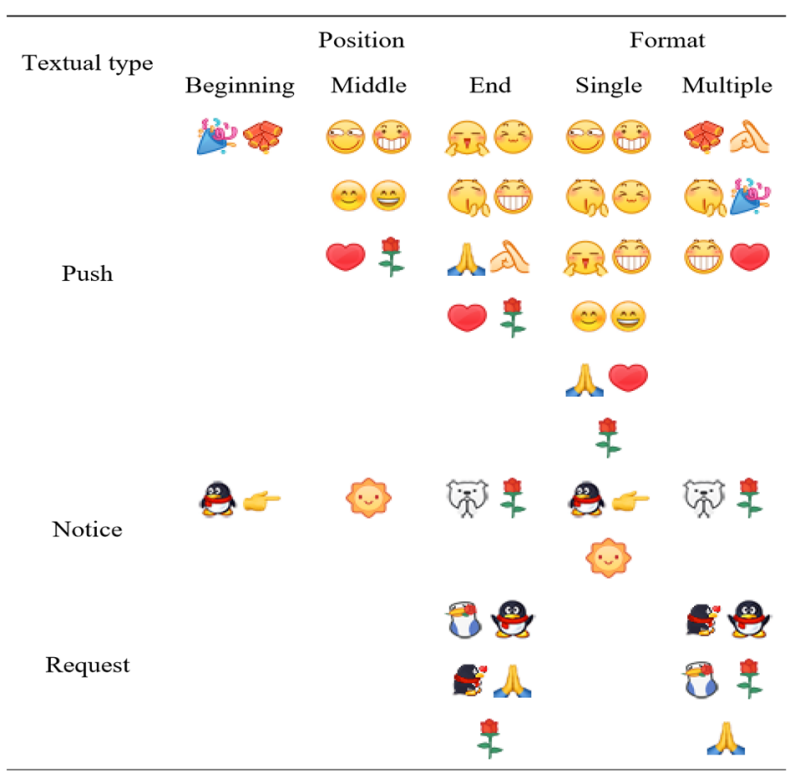

\section{DISCUSSION}

Emoji usage improved the quality of message in terms of voicing emotion, attracting attention and showing politeness. And the fostered playfulness [13] facilitated social network between senders and receivers. College students, therefore, were able to be socially persuasive by playing QQ emojis to initiate engagement in MMC.

\subsection{Emoji preference in play frame}

In fact, expression intensity mainly comes from users' preference. Users engage in a situation in search of emojis they have already seen or hear about, in order to deliver the same emotion by themselves. If they were asked to explain why, they would refer to their common ground to make sense of their participation. From person to person, the emoji becomes salient in certain of context. The higher use frequency an emoji enjoys, the stronger connection between the emoji and the type of emotion it represents. There might be a substitute, but peer pressure in speech community stops them from doing so simply because the substitute is not the one can comparably handle their entire feelings at that moment. Play frame in MMC, therefore, works all way through. And how users play emojis matters, it directs users' preference which further positively affect the list of popular emojis. As for emojis with strong expression intensity, they are instantiated stylistic patterns or linguistic features that repeatedly appear in the dialog boxes. For example, if 
initiators did not prefer "face with tear of joy", there will be another one to be compiled into Oxford Dictionaries Word of the Year 2015.

\subsection{Playfulness in pan-entertainment}

If play frame addresses what makes a good or popular emoji, playfulness explicates why users play emoji in MMC. The former focuses on the interior of emoji, while the latter looks at the exterior. Playfulness is prone to justify the joy of emoji usage, as what social psychologists defined it - a dispositional tendency to engage in play. Or rather, playing emoji helps users fulfil their expectations of having fun when taking part in online activities. Highly interactive in nature, playfulness also channels emoji to materialize some linguistic features as shown in 4.2. Those emojis function as an attention-getter at the beginning of message, visualize emotion flow at the transitional part and optimize the sincerity in the end.

However, emoji usage also blurs the boundaries between formality and informality in writing. Because the recreational function of emoji could melt the solemness and corrode the authority between the lines so as to build an equal and easy-going communicative atmosphere. More remarkably, the playfulness is hard to control in an internet community driven by panentertainment. And the amplified playfulness is subtle because play, the core of playfulness, is neither described important, nor a respectable medium for intervention. And those traditionally inappropriate presences of emoji in formal context become more and more cognitively acceptable after practices and practices.

\section{CONCLUSION}

Present study, led by a functional approach, emphasizes on emoji usage. Emotion conveyance, as the most fundamental service an emoji can provide, of which the expression intensity is hooked by use frequency. The appeal and curtesy functions in three forms of texts suggest viewing emoji socially. While the cause and effect of emoji usage is situated at the notion of play to some extent: how users play emojis matters. However, limitations accompany. The collected chatting records of three QQ groups spanned only half a year. And college students are only a small part of internet community, the results may not be shared with other emoji users.

\section{REFERENCES}

[1] C. B. Durante, Adapting Nonverbal Coding Theory to Mobile Mediated Communication: An Analysis of Emoji and Other Digital Nonverbals, Liberty University, (2016).
[2] P. K. Novak, J. Smailović, B. Sluban, I. Mozetič, Sentiment of Emojis, PLoS ONE, 10(12) (2015). DOI: http://10.1371/journal.pone.0144296

[3] A. Georgakopoulou, Postscript: Computermediated Communication in Sociolinguistics, Journal of Sociolinguistics, 10(4) (2006), 548-557.

[4] H. Miller, D. Kluver, J. Thebault-Spieker, L. Terveen, B. Hecht, Understanding Emoji Ambiguity in Context: The Role of Text in Emoji-related Miscommunication, in: Proceedings of the Eleventh International AAAI Conference on Web and Social Media, 11(1), 2017.

[5] P. Wicke, M. Bolognesi, Emoji-based Semantic Representations for Abstract and Concrete Concepts, Cognitive Processing, 21(4) (2020), 615-635. DOI: https://doi.org/10.1007/s10339-020-00971-x

[6] D. Marengo, F. Giannotta, M. Settanni, Assessing Personality Using Emoji: An Exploratory Study, Personality and Individual Differences, 112 (2017), 74-78.

[7] A. Beattie, A. Edwards, C. Edwards, A Bot and a Smile: Interpersonal Impressions of Chatbots and Humans Using Emoji in Computer-mediated Communication, Communication Studies 71(3) (2020) 409-427.

[8] M. Van Vleet, B. C. Feeney, Play Behavior and Playfulness in Adulthood, Social and Personality Psychology Compass, 9(11) (2015), 630-643. DOI: http://doi.org/10.1111/spc3.12205

[9] X. Wang, An Analysis of the Linguistic Form and Pan Entertainment Phenomenon of Cyberbullying Language, World Scientific Research Journal, 6(8) (2020), 16-23.

[10] M. Del-Teso-Craviotto, Language and Sexuality in Spanish and English Dating Chats, Journal of Sociolinguistics, 10(4) (2006), 460-480.

[11] P. Ekman, W.V. Friesen, The Repertoire of Nonverbal Behavior: Categories, Origins, Usage, and Coding, Semiotica, 1(1) (1969), 49-98.

[12] B. Liu, Y. Niu, J. Zhang, Statistical Analysis of Emoticons and Its Application in Emotion Analysis, Computer Engineering \& Science, 38(3) (2016), 577-584. DOI: http://10.3969/j.issn.1007130X.2016.03.027

[13] S. H. Hsieh, T. H. Tseng, Playfulness in Mobile Instant Messaging: Examining the Influence of Emoticons and Text Messaging on Social Interaction, Computers in Human Behavior, 69 (2017), 405-414. 